\title{
Electron collisions with CO molecule: An R-matrix study using a large basis set
}

\author{
Amar Dora ${ }^{1}$ and Jonathan Tennyson ${ }^{2}$ \\ 1 Department of Chemistry, North Orissa University, Baripada 757003, Odisha, \\ India, amardora@gmail.com \\ 2 Department of Physics and Astronomy, University College London, Gower St., \\ London WC1E 6BT, UK, j.tennyson@ucl.ac.uk
}

\begin{abstract}
Fixed-nuclei R-matrix calculations are performed at the equilibrium geometry of carbon monoxide using the very large cc-pV6Z Gaussian basis set. Results from a close-coupling model involving 27 low-lying target states indicate the presence of three ${ }^{2} \Sigma^{+}$resonances at $10.1 \mathrm{eV}$ (width $0.1 \mathrm{eV}), 10.38 \mathrm{eV}(0.0005 \mathrm{eV})$ and $11.15 \mathrm{eV}(0.005 \mathrm{eV}), \mathrm{a}^{2} \Delta$ resonance at $13.3 \mathrm{eV}(0.1 \mathrm{eV})$ and two ${ }^{2} \Pi$ resonances at $1.9 \mathrm{eV}(1.3$ $\mathrm{eV})$ and $12.8 \mathrm{eV}(0.1 \mathrm{eV})$. These new results are in very good agreement with many experimental studies but in contrast to a previous calculation using a smaller cc-pVTZ basis set where we found only one ${ }^{2} \Sigma^{+}$resonances at $12.9 \mathrm{eV}$. This is the first time that any theoretical study has reported these high lying ${ }^{2} \Sigma^{+}$resonances in agreement to experiment and reported detection of $\mathrm{a}^{2} \Delta$ resonance. Total, elastic and electronic excitation cross sections of $\mathrm{CO}$ by electron impact are also presented.
\end{abstract}

Keywords: Carbon monoxide, Resonances, R-matrix method

\section{Introduction}

The study of various processes involving collision of electrons with molecules is of fundamental interest to many areas of science and technology. The cross sections of these processes get significantly enhanced in certain narrow collision energy range due to the formation of metastable anionic states, called resonances, which are formed due to temporary capture of the colliding electron by the molecule. Therefore, the resonances play an important role in processes such as dissociative electron attachment (DEA) and their detection and characterization is a major part of any electron-molecule collision study.

Resonances in low-energy electron collision with the $\mathrm{CO}$ molecule have been studied by several groups in the past. A classic review of resonances in CO, along with other diatomic molecule, was given by Schulz [1]. In addition, a compilation of available cross sections of different processes in electron-CO collisions is given by Itikawa [2]. Most of the works on $\mathrm{CO}$ have been directed towards the lowest lying and well-known ${ }^{2} \Pi$ resonance at $1.6 \mathrm{eV}$ and on the elastic and vibrational excitation processes associated with it. In the low and intermediate energy range there have been quite a few experimental [3-6] and theoretical [7-11] studies 
on electron impact electronic excitation of CO. However, there is very little consensus among them on the presence and characteristics of the higher-lying resonances.

Electron collision with CO can produce anionic fragments through the DEA process as both $\mathrm{C}$ and $\mathrm{O}$ can form stable anions. Formation of these atomic anions as result of electron collisions has indeed been observed for a long time and their production cross sections has been measured by several groups [1217]. These cross sections show a broad peak in the 9 to $12 \mathrm{eV}$ energy range. The presence of a ${ }^{2} \Sigma^{+}$resonance at $10.04 \mathrm{eV}$ has been established by Sanche and Schulz [18] and is thought to contribute to this DEA peak. Furthermore, shape resonances at $10.4 \mathrm{eV}$ and $10.7 \mathrm{eV}$ and Feshbach resonances at $11.3 \mathrm{eV}$ and 12.2 $\mathrm{eV}$ were reported from experiments $[1,18,19]$; these may also contribute to DEA.

In the DEA process $\mathrm{O}^{-}$ion production is favored and the cross section of $\mathrm{C}^{-}$ion is very weak. Therefore, recently there been attempts by experimentalists $[17,20,21]$ to establish the nature of resonances through the measurement of $\mathrm{O}^{-}$angular distributions. However, these groups arrived at different conclusions from the analysis of their results while using essentially the same experimental technique of velocity time sliced imaging method. Newer and more precise measurements made by Gope et al. [22] have helped to settle the above controversy $[23,24]$, but the problem calls for accurate theoretical calculations.

In our recent attempt [25] to study the higher lying resonances in CO, our best model, close-coupling method with 50 target states represented using a ccpVTZ basis set, found only one ${ }^{2} \Sigma^{+}$resonance at $12.9 \mathrm{eV}$ in addition to the lowlying ${ }^{2} \Pi$ at $1.7 \mathrm{eV}$. In this study, we report on a similar fixed-nuclei calculation but with a much larger cc-pV6Z basis set. Here, we find clear signatures of a number of narrow resonances lying in the 10 to $13 \mathrm{eV}$ range that are in great agreement with previous experimental findings.

\section{Theory}

In this section, we describe the theory briefly. Details of the $\mathbf{R}$-matrix method and its implementation in the UK molecular R-matrix codes $[26,27]$ can be found in the review article by Tennyson [28].

The R-matrix method, which is employed here, divides the space around the electron+target system into an inner region, inside which the exchange and correlation effects among all $N+1$ electrons is explicitly considered, and an outer region, where the scattering electron is considered to be interacting with the multipolar potential of the $N$-electron target. In the inner region the scattering wavefunction, $\psi_{k}^{N+1}$, is represented using a close-coupling (CC) expansion:

$$
\psi_{k}^{N+1}=\mathcal{A} \sum_{i j} a_{i j k} \Phi_{i}^{N}\left(\mathbf{x}_{1} \ldots \mathbf{x}_{N}\right) u_{i j}\left(\mathbf{x}_{N+1}\right)+\sum_{i} b_{i k} \chi_{i}^{N+1}\left(\mathbf{x}_{1} \ldots \mathbf{x}_{N+1}\right),
$$

where $\mathbf{x}_{i}$ are the space-spin coordinates of the electrons. The $\Phi_{i}^{N}$ in Eq. (1) represents the wavefunction of the $i$-th target state, the $u_{i j}$ are the continuum 
orbitals representing the scattering electron and $\mathcal{A}$ is the anti-symmetrization operator. The $\chi_{i}^{N+1}$, in the second term, are called $L^{2}$ configurations. These configurations are constructed by occupation of the target molecular orbitals (MOs) by all $N+1$ electrons. The $a_{i j k}$ and $b_{i k}$ are variational parameters which are obtained from the diagonalization of the scattering Hamiltonian [29].

At the boundary of the inner region sphere, the R-matrix is calculated from the boundary amplitude of the inner region wavefunctions and the R-matrix poles. The R-matrix is then propagated outwards and matched to analytical asymptotic scattering functions. From this matching the K-matrix is calculated as a function of scattering energy. The K-matrix is a key quantity and other scattering observables can be obtained from this.

In the following section we use two quantities, the eigenphase sum and the time-delay, to detect and fit the resonances. The fitting of eigenphase sum to the Breit-Wigner form yields the resonance position and width, which is done automatically by the module RESON [30] in the UKRmol codes. On the other hand, the time-delay is fitted to a Lorentian function through the module TIMEDEL $[31,32]$ to get the same resonance parameters.

\section{Calculation and Results}

As in the previous paper [25] (hereafter referred as paper-I), here we report on $\mathbf{R}$-matrix calculation at the equilibrium geometry of $\mathrm{CO}, R_{e q}=2.1323 \mathrm{a}_{0}$. As mentioned above, we use the UK molecular R-matrix codes (also called UKRmol codes) $[26,27,33]$ for the scattering calculations. The necessary target molecular orbitals are obtained from MOLPRO [34] using the largest supported cc-pV6Z basis set which has no augmented diffused functions. The calculations were performed in the $\mathrm{C}_{2 v}$ point group since neither MOLPRO nor the Gaussian version of the UKRmol codes can use the full $\mathrm{C}_{\infty v}$ symmetry of $\mathrm{CO}$ molecule. However, the target and resonant states in the $\mathrm{C}_{2 v}$ symmetry can be clearly correlated with their $\mathrm{C}_{\infty v}$ counterparts. Therefore, these states are reported below in $\mathrm{C}_{\infty v}$ symmetry.

\subsection{Target results}

The target molecular orbitals necessary in $\mathbf{R}$-matrix calculations are obtained from MOLPRO using the state-averaged complete active space (CAS) self-consistentfield (SCF) method. We used the LQUANT option available in MOLPRO CASSCF program to specify the $L_{z}$ quantum number and hence obtained the target states in $C_{\infty v v}$ symmetry. A total of 27 low lying target states in $C_{\infty v}$ symmetry were computed. These are: $4{ }^{1} \Sigma^{+}, 2{ }^{1} \Sigma^{-}, 5^{1} \Pi, 2{ }^{1} \Delta, 4{ }^{3} \Sigma^{+}, 3{ }^{3} \Sigma^{-}, 5^{3} \Pi$ and $2^{3} \Delta$. In terms of $\mathrm{C}_{2 v}$ assignments this amounts to 41 target states once the degenerate states of $\Pi$ and $\Delta$ symmetry states are accounted for. The CASSCF active space is the same as in paper-I, where 10 valence electrons are distributed freely over 10 valence orbitals. The active space configuration is defined as: $\left(1 a_{1}-2 a_{1}\right)^{4}\left(3 a_{1}-6 a_{1}, 1 b_{1}-3 b_{1}, 1 b_{2}-3 b_{2}\right)^{10}$. 
Table 1. The CASSCF ground state energy (in $\mathrm{E}_{h}$ ), the lowest 11 vertical excitation energies (in $\mathrm{eV}$ ) and ground state dipole moments ( $\mu$ in $\mathrm{D}$ ) of $\mathrm{CO}$ calculated using cc-pV6Z basis set. The target results using cc-pVTZ basis set are also included for comparison. The experimental data are from Nielsen et al.[35]

\begin{tabular}{cccc}
\hline State & cc-pVTZ & cc-pV6Z & Expt \\
\hline$X^{1} \Sigma^{+}$ & -112.8565508 & -112.8599842 & \\
$1^{3} \Pi$ & 6.31 & 6.43 & $6.32\left(a^{3} \Pi\right)$ \\
$1^{3} \Sigma^{+}$ & 8.39 & 8.36 & $8.51\left(a^{\prime 3} \Sigma^{+}\right)$ \\
$1^{1} \Pi$ & 8.83 & 8.97 & $8.51\left(A^{1} \Pi\right)$ \\
$1^{3} \Delta$ & 9.23 & 9.22 & $9.36\left(d^{3} \Delta\right)$ \\
$1^{3} \Sigma^{-}$ & 9.60 & 9.60 & $9.88\left(e^{3} \Sigma^{-}\right)$ \\
$1^{1} \Sigma^{-}$ & 9.97 & 9.95 & $9.88\left(I^{1} \Sigma^{-}\right)$ \\
$1^{1} \Delta$ & 10.00 & 10.00 & $10.23\left(D^{1} \Delta\right)$ \\
$2^{3} \Sigma^{+}$ & 12.90 & 10.39 & $10.40\left(b^{3} \Sigma^{+}\right)$ \\
$2^{1} \Sigma^{+}$ & 13.76 & 11.16 & $10.78\left(B^{1} \Sigma^{+}\right)$ \\
$2^{3} \Pi$ & 12.29 & 11.34 & \\
$2^{1} \Pi$ & 13.72 & 11.84 & \\
$\mu$ & 0.291 & 0.238 & 0.122 \\
\hline
\end{tabular}

Table 1 presents the target ground state energy (in $E_{h}$ ), vertical excitation energies to lowest 11 states (in $\mathrm{eV}$ ) and the ground state dipole moment (in Debye) for the cc-pV6Z basis set. It also includes the target results from paper-I obtained with cc-pVTZ basis set along with the experimental values as reported by Nielsen et al.[35] for the purpose of comparison. As can be seen the in the Table the first seven excitation energies in both cc-pVTZ and cc-pV6Z basis sets are in excellent agreement with each other and also to the experimental values. However, if we compare the $2^{3} \Sigma^{+}$state, which is designated as $b^{3} \Sigma^{+}$in spectroscopic notation, the CASSCF value of $10.39 \mathrm{eV}$ with cc-pV6Z basis set is very close to the experimentally determined value of $10.4 \mathrm{eV}$, while that in case of cc-pVTZ basis set is $12.9 \mathrm{eV}$. Similarly, the energy for $2^{1} \Sigma^{+}$state from the cc-pV6Z basis set is in much better agreement with experiment than that from the cc-pVTZ basis set. Notwithstanding the above argument, it may be pointed out that the vertical excitation energies of the first four target states obtained using the much lower cc-pVTZ basis set are slightly closer to the experimentally estimated values than those from the larger cc-pV6Z basis set. This might happen given that the excitation energies are obtained by finding the difference between the absolute energies of the ground state and the excited states. Of course, the absolute energies of all the states calculated with cc-pV6Z basis set are smaller than those calculated with the cc-pVTZ basis set, as to be expected from a variational method. Moreover, the theoretically calculated ground state dipole moment of $\mathrm{CO}$ is twice that of the experimental value despite use of the large cc-pV6Z basis set. We assign this difference to our use of the CASSCF method. In order to obtain accurate dipole moments close to the experiments, one would need to calculate highly correlated wavefunctions as obtained in multi-reference 
configuration interaction methods. However, we did not pursue this aspect as it was not the purpose of this work.

In the $\mathbf{R}$-matrix calculations the relative energy positions of different excited states with respect to the ground state is very important. Since resonances are often closely associated with a parent target state [36], getting correct target positions is of tremendous importance to get resonance positions correctly. In the paper-I we found a very narrow Feshbach resonance lying extremely close to the $2^{3} \Sigma^{+}$target state at $12.9 \mathrm{eV}$ and therefore we assigned the later to be its parent. In the present calculation we get this target state very close to the experimental value of $10.4 \mathrm{eV}$, therefore we expect this resonance to be near to $10.4 \mathrm{eV}$. It is known from experiments that the $b^{3} \Sigma^{+}\left(2^{3} \Sigma^{+}\right)$state of CO has Rydberg character. In the present calculation with cc-pV6Z basis set, which includes higher angular momentum basis functions upto i-functions $(l=6)$, the Rydberg nature of the state is represented correctly.

\subsection{Scattering results}

Table 2. Positions (and widths) of the resonances as detected by the RESON module in fitting of eigenphase sums and by the TIMEDEL module in fitting of the time-delays. All quantities are in $\mathrm{eV}$.

\begin{tabular}{ccc}
\hline Symmetry & RESON & TIMEDEL \\
\hline${ }^{2} \mathrm{~A}_{1}$ & $10.1019(0.1126)$ & $10.0924(0.1277)$ \\
& $10.3850(0.00049)$ & - \\
& $11.1579(0.0048)$ & $11.1576(0.0047)$ \\
& $13.2969(0.0977)$ & $13.2916(0.1310)$ \\
${ }^{2} \mathrm{~A}_{2}$ & $13.3135(0.1641)$ & $13.2823(0.1456)$ \\
${ }^{2} \mathrm{~B}_{1}$ & $1.8744(1.2916)$ & - \\
& $12.8306(0.0889)$ & $12.8302(0.0891)$ \\
\hline
\end{tabular}

In the following we present the results obtained from the close-coupling (CC) scattering calculation using the above mentioned 27 target states. Here, we do not explore on calculations with different scattering models such as the staticexchange and static-exchange with polarization models, as these are unsuitable for describing the higher lying resonances that are associated with excited target states. Such studies has been already performed previously in paper-I where extensive testing with respect to basis sets, molecular orbitals, $\mathbf{R}$-matrix radius, etc. has been performed.

In the present $\mathrm{CC}$ calculation an $\mathbf{R}$-matrix sphere of radius $a=12 a_{0}$ is used and the continuum functions, appropriate for this radius [37], using partial waves upto $\ell \leq 4$ are included. The necessary occupied and virtual target molecular orbitals are obtained from MOLPRO by doing the state-averaged CASSCF calculation for the 27 target states which carry equal weights. 
The positions and widths of the resonances that are found in this study are listed in Table 2. We have used both the eigenphase sum and the time-delay to find the resonance parameters. Table 2 contains the fitted resonance parameters from both RESON and TIMEDEL programs. As can be seen in the Table both the methods give almost identical values in most of the cases. To show the resonances obtained in the calculation we provide the eigenphase sum and the time-delay plots as a function of scattering energy.

Figure 1 shows the eigenphase sum and the time-delay for the ${ }^{2} \mathrm{~B}_{1}$ symmetry. ${ }^{2} \mathrm{~B}_{1}$ and ${ }^{2} \mathrm{~B}_{2}$ symmetry results are identical as these constitute the two degenerate parts of the ${ }^{2} \Pi$ symmetry. The lowest lying ${ }^{2} \Pi$ resonance is well-known from many experiments and theoretical calculations. In our calculation the position of this resonance is found by RESON to be at $1.87 \mathrm{eV}$ with a width of $1.29 \mathrm{eV}$, while TIMEDEL missed this resonance. Most of the experiments report the position of this resonance to be at $1.6 \mathrm{eV}$. It is worth pointing here that in our previous work using the cc-pVTZ basis set we obtained this resonance at $1.73 \mathrm{eV}$, which is lower than the present value and closer to the experimentally reported data. We can explain this on the basis that the $\mathbf{R}$-matrix method relies on the difference between the target and scattering states energies. Although each part is variational and gives lower absolute energies for both the target and scattering states, the difference between them can lead to resonances moving to higher energy as the basis set is improved. This behaviour has also been observed in other studies [38]. In addition to the lowest one we find a higher energy ${ }^{2} \Pi$ resonance lying at $12.83 \mathrm{eV}$ with a narrow width of $0.1 \mathrm{eV}$. This can be seen in both the eigenphase sum and the time-delay plots.

The total and elastic cross sections from the ${ }^{2} \Pi\left({ }^{2} \mathrm{~B}_{1}+{ }^{2} \mathrm{~B}_{2}\right)$ symmetry is presented in Figure 2 and the dominant electron impact electronic excitation cross sections in Figure 3. These figures show the effect of the ${ }^{2} \Pi$ resonances on different cross sections. The lowest ${ }^{2} \Pi$ resonance causes a huge increase in the total and elastic cross section while the higher ${ }^{2} \Pi$ resonance has very little effect on these cross sections.

The eigenphase sum and the time-delay plots for the ${ }^{2} \mathrm{~A}_{1}$ and ${ }^{2} \mathrm{~A}_{2}$ symmetries are shown together in Figure 4. For the ${ }^{2} \mathrm{~A}_{1}$ symmetry we find four resonances at $10.1 \mathrm{eV}$ (width $0.1 \mathrm{eV}$ ), $10.385 \mathrm{eV}$ (width $0.00048 \mathrm{eV}$ ), $11.158 \mathrm{eV}$ (width 0.0048 $\mathrm{eV})$ and $13.29 \mathrm{eV}(0.1 \mathrm{eV})$; while for the ${ }^{2} \mathrm{~A}_{2}$ symmetry we find a single resonance at around $13.3 \mathrm{eV}$ (width $0.1 \mathrm{eV}$ ). Both the ${ }^{2} \mathrm{~A}_{1}$ and ${ }^{2} \mathrm{~A}_{2}$ scattering calculations find a common resonance at around $13.3 \mathrm{eV}$ with a width of approximately 0.1 $\mathrm{eV}$. Therefore, we assign them to be the two components of a ${ }^{2} \Delta$ resonance. The first three ${ }^{2} A_{1}$ resonances at $10.1 \mathrm{eV}, 10.385 \mathrm{eV}$ and $11.158 \mathrm{eV}$ are assigned to be of ${ }^{2} \Sigma^{+}$symmetry in the natural $C_{\infty v}$ symmetry of $\mathrm{CO}$ molecule. The second and third ${ }^{2} \Sigma^{+}$resonances are very narrow and lie extremely close to the $2^{3} \Sigma^{+}$ and $2{ }^{1} \Sigma^{+}$target states, respectively. We assign these target states to be their respective parents. With this the binding energy of the second ${ }^{2} \Sigma^{+}$resonance with respect to its parent $2^{3} \Sigma^{+}$state would be $0.001 \mathrm{eV}$, while that of the third ${ }^{2} \Sigma^{+}$resonance with respect to its parent $2{ }^{1} \Sigma^{+}$state would be $0.006 \mathrm{eV}$. All 

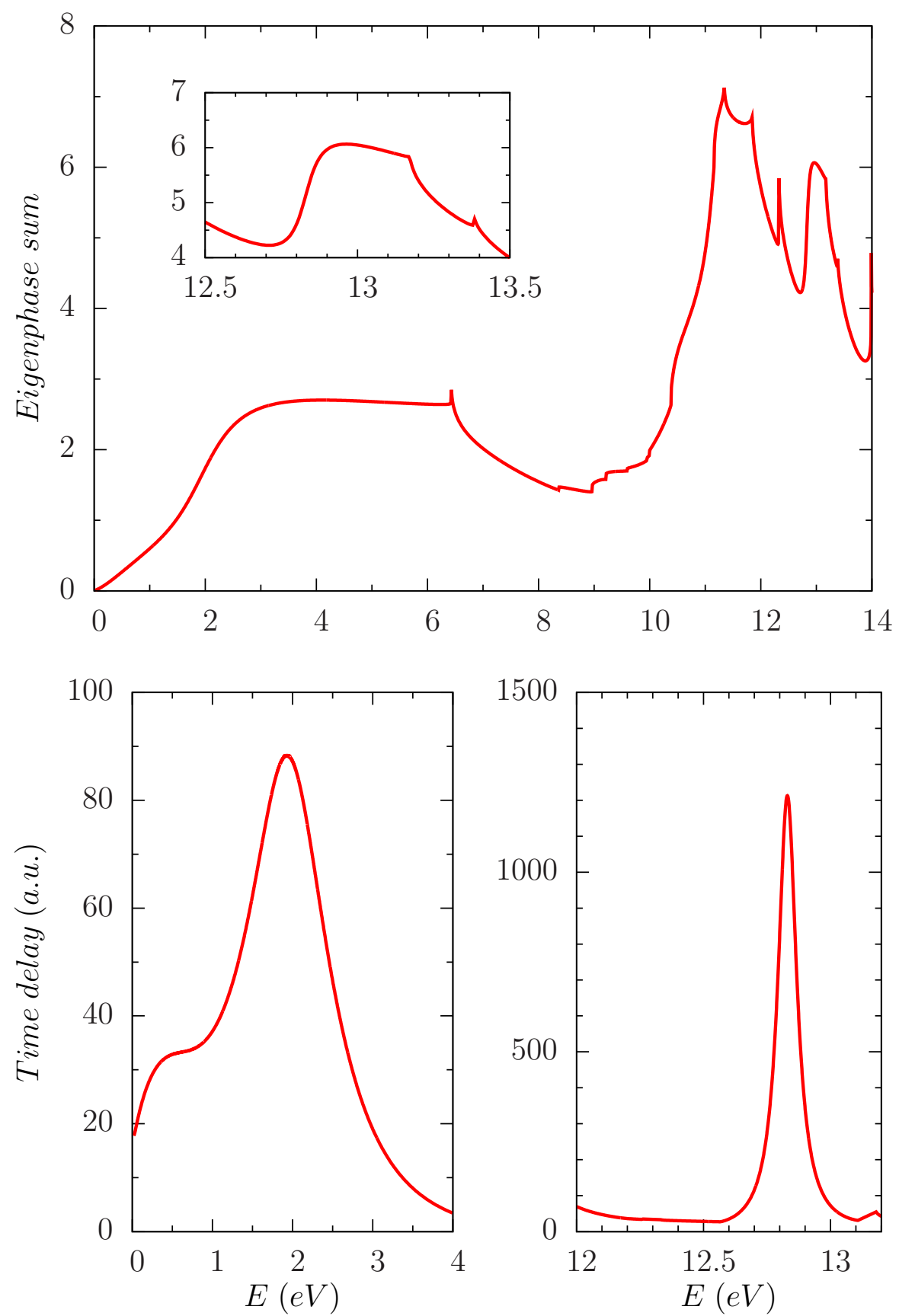

Fig. 1. ${ }^{2} \mathrm{~B}_{1}$ symmetry: the upper panel shows the eigenphase sum and the lower shows the time-delay as a function of scattering energy. The inset in the upper panel clearly shows the second ${ }^{2} \Pi$ resonance at $12.83 \mathrm{eV}$. 


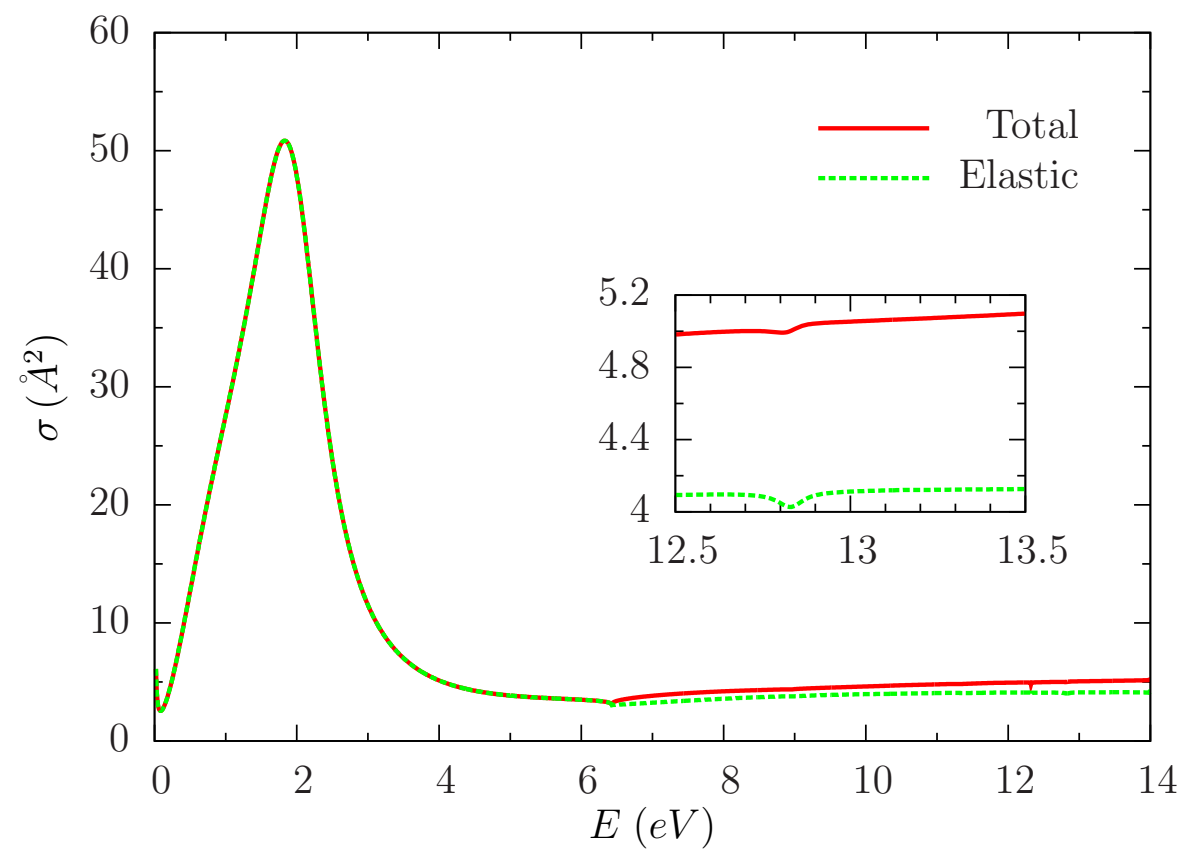

Fig. 2. Total and elastic cross sections for ${ }^{2} \mathrm{~B}_{1}+{ }^{2} \mathrm{~B}_{2}\left({ }^{2} \Pi\right)$ symmetry. The inset shows the effect of the second ${ }^{2} \Pi$ resonance on the cross sections clearly. 


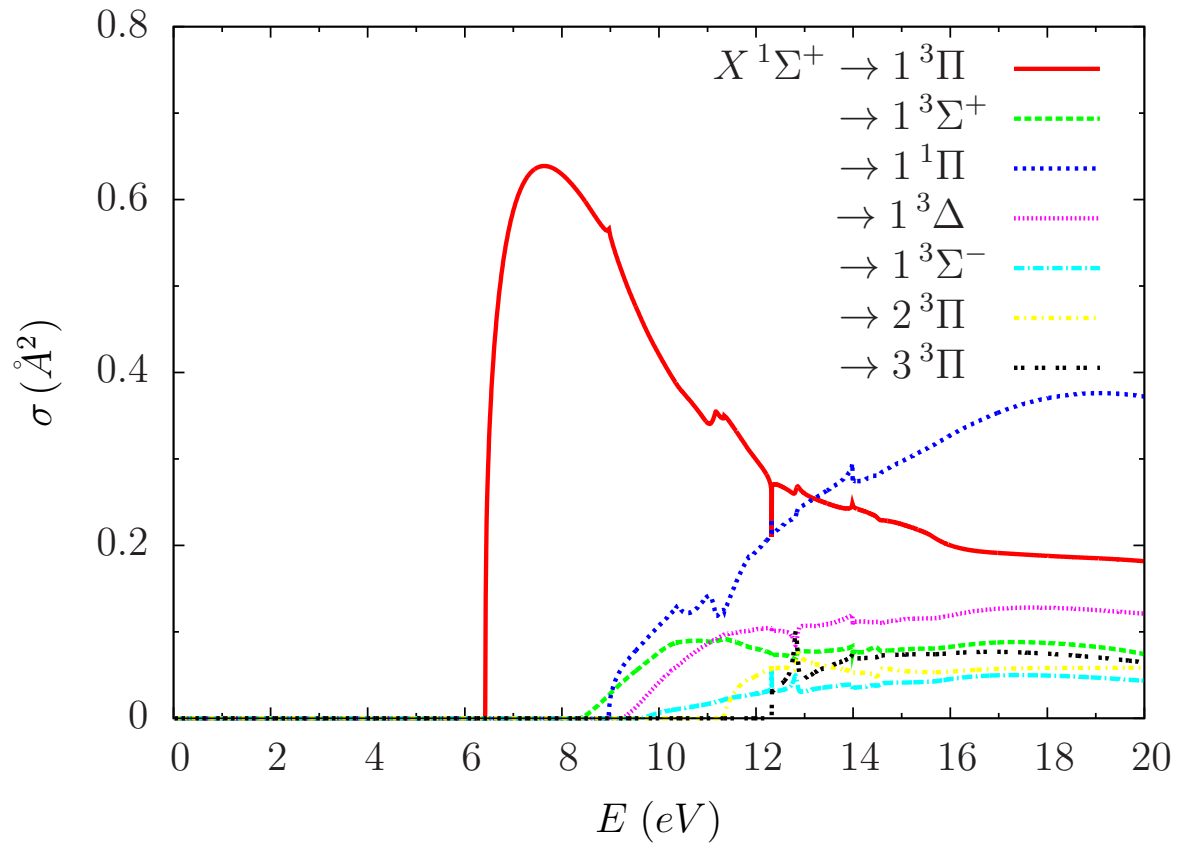

Fig. 3. The dominant electron impact excitation cross sections for ${ }^{2} \mathrm{~B}_{1}+{ }^{2} \mathrm{~B}_{2}\left({ }^{2} \Pi\right)$ symmetry. 

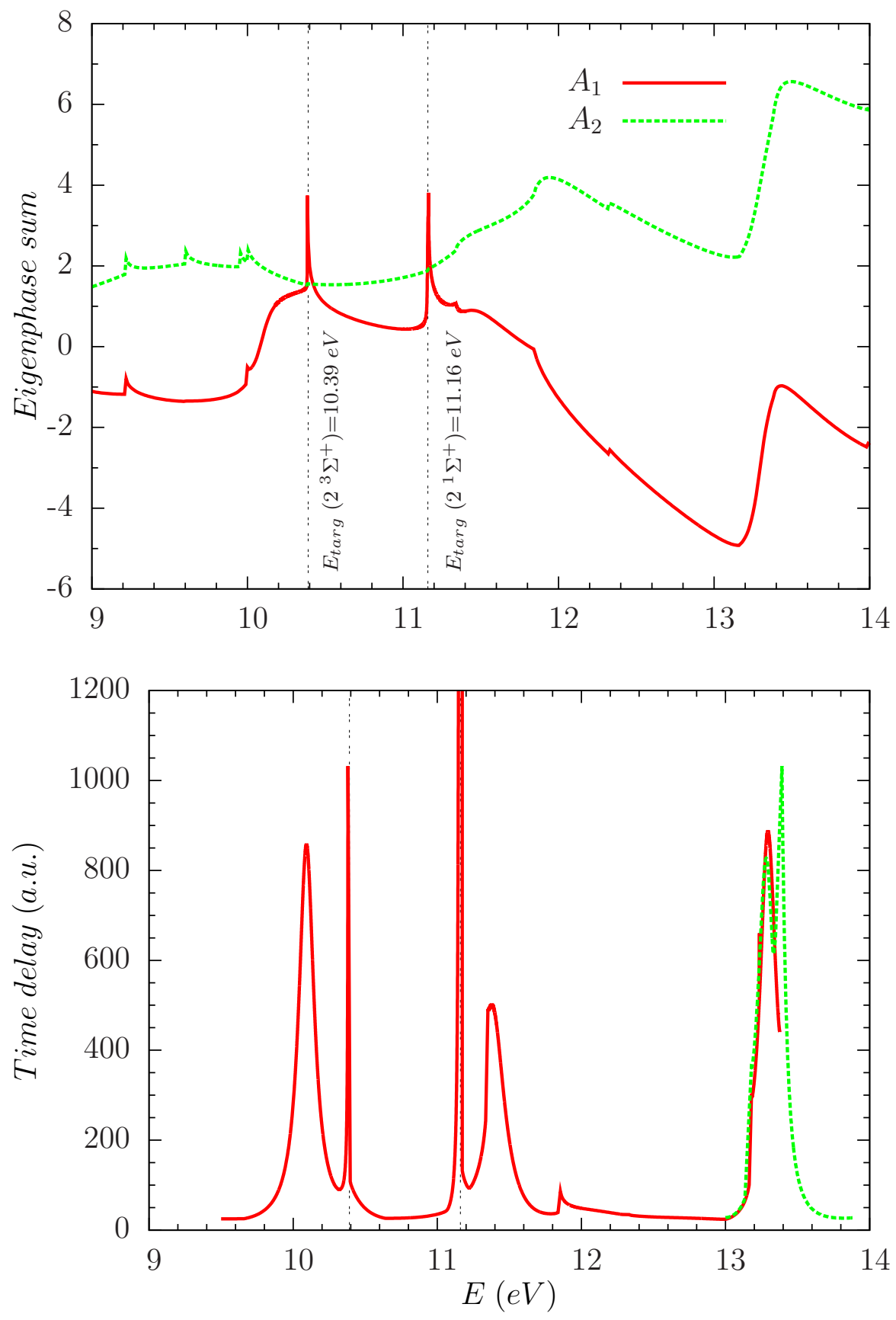

Fig. 4. Eigenphase sum (upper) and time-delay (lower) plots for both ${ }^{2} \mathrm{~A}_{1}$ and ${ }^{2} \mathrm{~A}_{2}$ symmetries. The vertical dashed lines indicate the excitation thresholds for the $2^{3} \Sigma^{+}$ and $2^{1} \Sigma^{+}$target states. 
these resonances are clearly seen in the Figure 4 where we have also marked the parent target states.

The effect of the ${ }^{2} \Sigma^{+}$and ${ }^{2} \Delta$ resonances can be seen in the total and elastic cross sections shown for ${ }^{2} \mathrm{~A}_{1}$ symmetry in Figure 5 . The dominant electron impact excitation cross sections for ${ }^{2} \mathrm{~A}_{1}$ symmetry is shown in Figure 6. Similarly, the Figure 7 shows the total and elastic cross sections, and Figure 8 shows the dominant electron impact excitation cross sections of $\mathrm{CO}$ in the ${ }^{2} \mathrm{~A}_{2}$ symmetry.

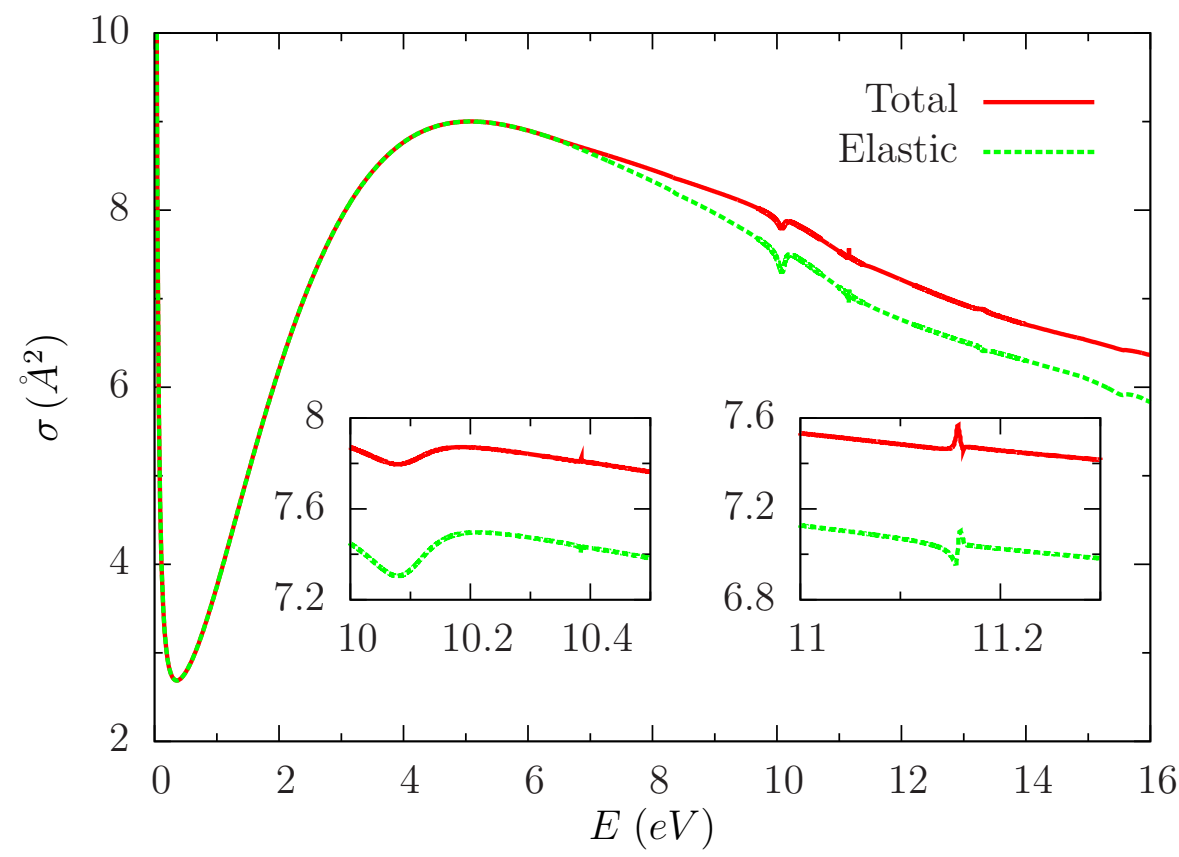

Fig. 5. Total and elastic cross section for ${ }^{2} \mathrm{~A}_{1}$ symmetry. The insets show the effect of the resonances on the cross sections.

\section{Conclusion}

We have undertaken an R-matrix study using a very large basis set of cc-pV6Z to identify higher energy resonances in electron scattering with carbon monoxide molecule. Our fixed-nuclei calculation at the equilibrium geometry of CO indicate the presence of three ${ }^{2} \Sigma^{+}$resonances, one ${ }^{2} \Delta$ resonance and one ${ }^{2} \Pi$ resonance in the energy range of $10 \mathrm{eV}$ to $14 \mathrm{eV}$, in addition to the low-lying ${ }^{2} \Pi$ resonance near $2 \mathrm{eV}$. The presence of a number of resonances above $10 \mathrm{eV}$ has been reported by many experiments, however, this is the first theoretical study to identify them clearly. The large cc-pV6Z basis set employed here effectively represents the Rydberg like target states of $\mathrm{CO}$ and therefore helps in getting the 


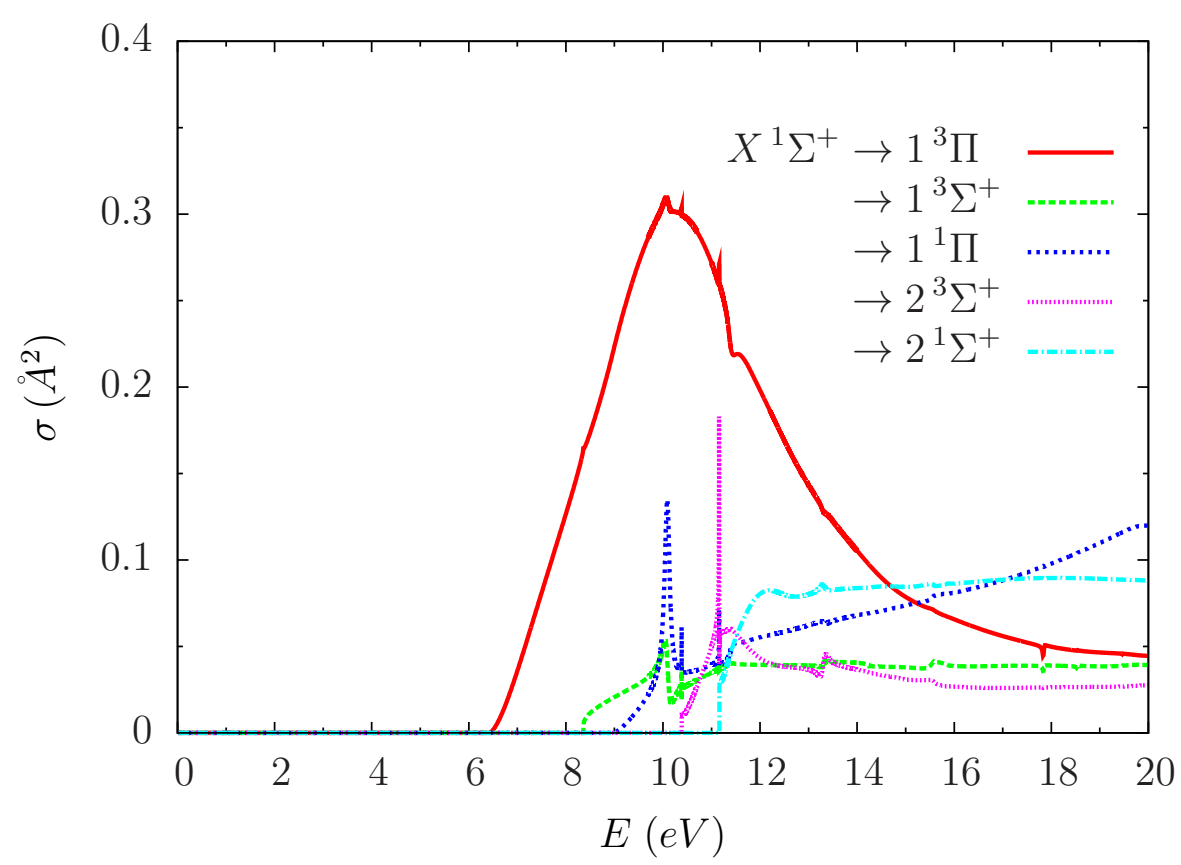

Fig. 6. The dominant electron impact excitation cross sections for ${ }^{2} \mathrm{~A}_{1}$ symmetry.

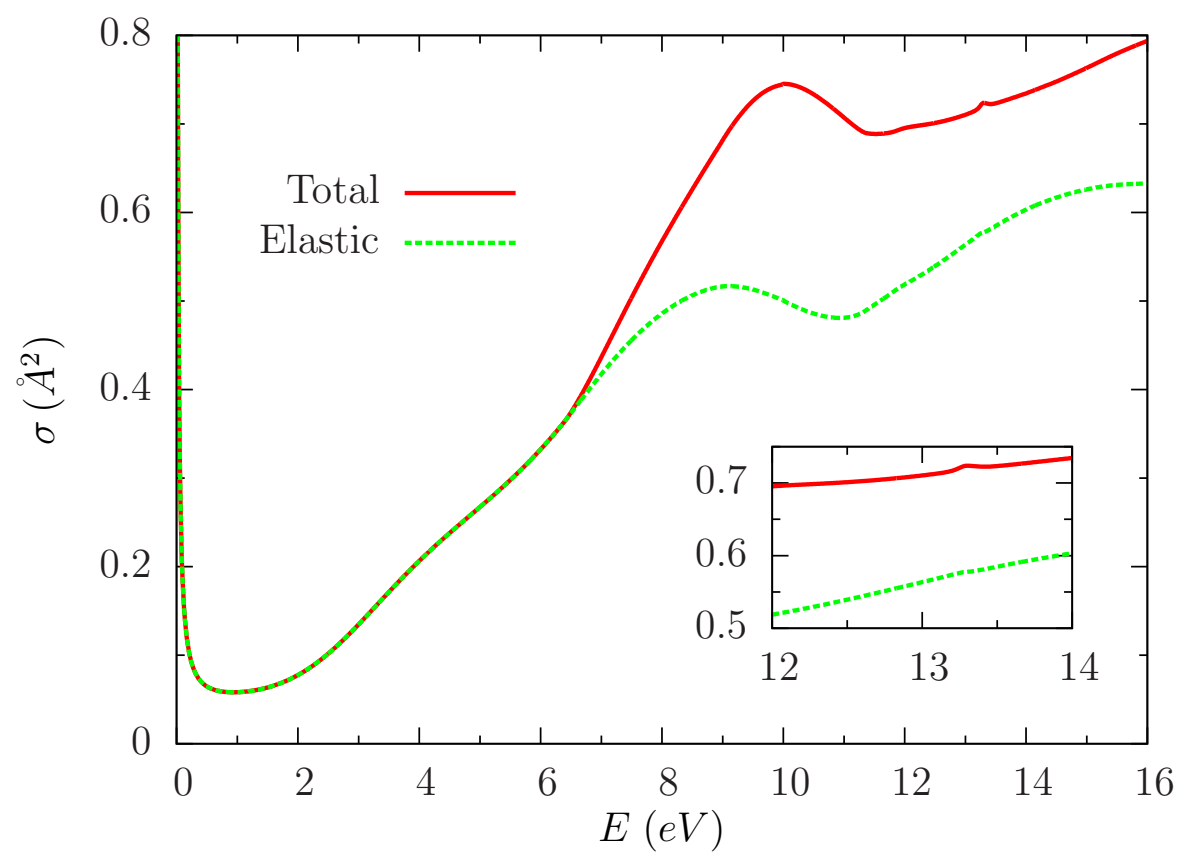

Fig. 7. Total and elastic cross sections of $\mathrm{CO}$ in the ${ }^{2} \mathrm{~A}_{2}$ symmetry. 


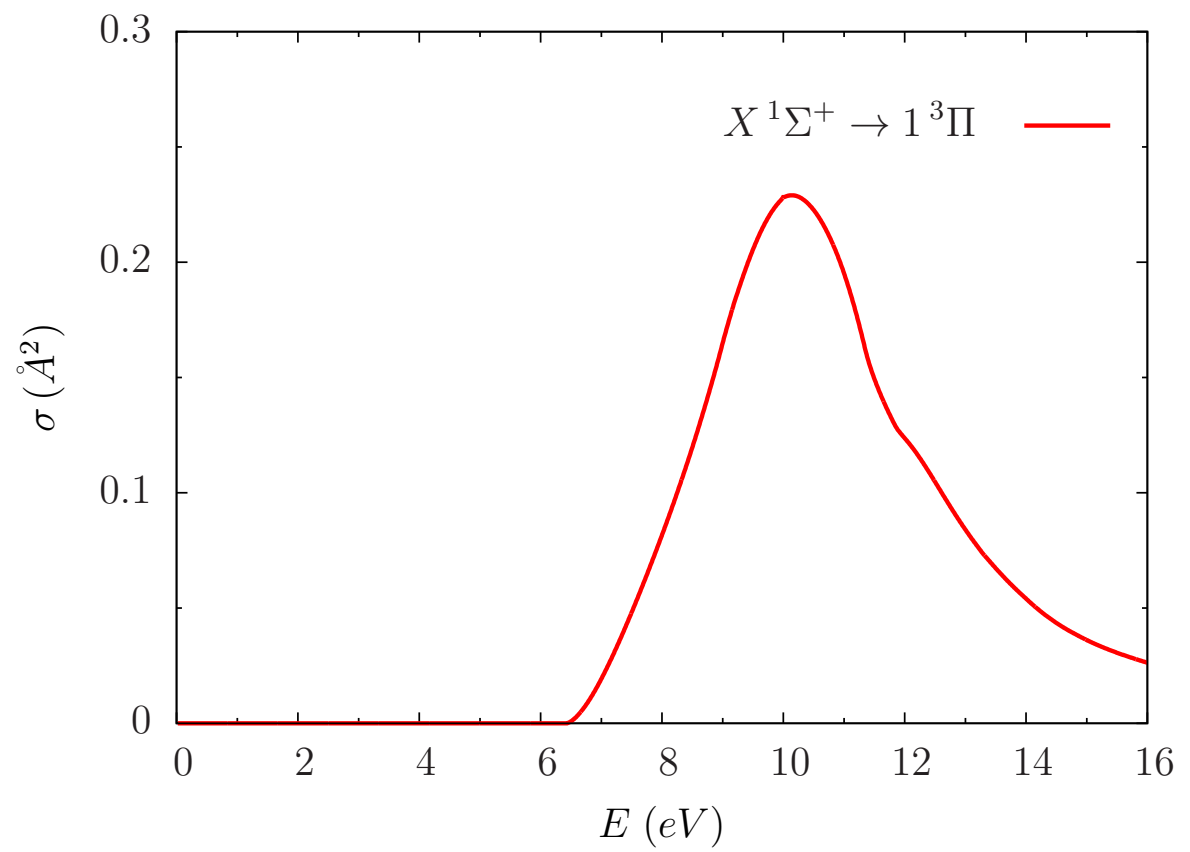

Fig. 8. The dominant electron impact excitation cross sections of $\mathrm{CO}$ in the ${ }^{2} \mathrm{~A}_{2}$ symmetry. 
Rydberg resonances in CO correctly. All these high energy resonances have very narrow widths and therefore can have longer lifetimes to result in dissociation giving anionic fragments. It is our purpose to study the DEA process from these resonances and we are already computing resonance parameters as a function of internuclear distance.

The work clearly shows the need to use extended basis sets to accurately represent both highly-excited target states and the associated resonances. This presents something of a challenge to R-matrix calculations due to the requirement that the target wavefunction must be entirely enclosed by the R-matrix sphere. However, we note the development of a new UK R-matrix code based on the use of B-splines for the continuum basis [39] which facilitates the uses of greatly extended inner regions.

\section{Acknowledgment}

AD gratefully acknowledges UCL for the use of its computational resources.

\section{References}

1. G.J. Schulz, Rev. Mod. Phys. 45, 423 (1973). DOI 10.1103/RevModPhys.45.423

2. Y. Itikawa, J. Phys. Chem. Ref. Data 44, 013105 (2015). DOI 10.1063/1.4913926

3. V.V. Skubenich, Optics and Spectroscopy 23, 540 (1967)

4. D.S. Newman, M. Zubek, G.C. King, J. Phys. B: At. Mol. Opt. Phys. 16, 2247 (1983). DOI https://doi.org/10.1088/0022-3700/16/12/019

5. J.P. Polley, T.L. Bailey, Phys. Rev. A 37, 733 (1988). DOI 10.1103/PhysRevA.37. 733

6. A.G. Middleton, M.J. Brunger, P.J.O. Teubner, J. Phys. B: At. Mol. Opt. Phys. 26, 1743 (1993). DOI 10.1088/0953-4075/26/11/009

7. C.A. Weatherford, W.M. Huo, Phys. Rev. A 41, 186 (1990). DOI 10.1103/ PhysRevA.41.186

8. L.A. Morgan, J. Tennyson, J. Phys. B: At. Mol. Opt. Phys. 26, 2429 (1993)

9. M.T. Lee, I. Iga, L.M. Brescansin, L.E. Machado, F.B.C. Machado, J. Molec. Struct. (THEOCHEM) 585, 181 (2002). DOI 10.1016/S0166-1280(02)00045-3

10. M. Gracas, R. Martins, A.M. Maniero, L.E. Machado, J.D.M. Vianna, Brazilian J. Phys 35, 945 (2005)

11. R. Riahi, P. Teulet, N. Jaidane, A. Gleizes, Eur. Phys. J. D 56, 67 (2010). DOI 10.1140/epjd/e2009-00267-5

12. D. Rapp, D.D. Briglia, J. Chem. Phys. 43, 1480 (1965). DOI 10.1063/1.1696958

13. P.J. Chantry, Phys. Rev. 172, 125 (1968)

14. A. Stamatovic, G.J. Schulz, J. Chem. Phys. 53, 2663 (1970). DOI 10.1063/1. 1674387

15. I. Cadex, M. Tronc, R.I. Hall, J. Phys. B: At. Mol. Opt. Phys. 8, L73 (1975). DOI 10.1088/0022-3700/8/5/003

16. R.I. Hall, I. Cadex, C. Schermann, M. Tronc, Phys. Rev. A 15, 599 (1977). DOI 10.1103/PhysRevA.15.599

17. P. Nag, D. Nandi, Phys. Chem. Chem. Phys. 17, 7130 (2015). DOI 10.1039/ c4cp05678g 
18. L. Sanche, G.J. Schulz, Phys. Rev. Lett. 26, 943 (1971). DOI 10.1103/ PhysRevLett.26.943

19. J. Mazeau, F. Gresteau, G. Joyez, J. Reinhardt, R.I. Hall, J. Phys.B: At. Mol. Phys. 5, 1890 (1972). DOI https://doi.org/10.1088/0022-3700/5/10/018

20. S.X. Tian, B. Wu, L. Xia, Y.F. Wang, H.K. Li, X.J. Zeng, Y. Luo, J. Yang, Phys. Rev. A 88, 012708 (2013). DOI 10.1103/PhysRevA.88.012708

21. X.D. Wang, C.J. Xuan, Y. Luo, S.X. Tian, J. Chem. Phys. 143, 066101 (2015). DOI 10.1063/1.4928639

22. K. Gope, V. Tadsare, V.S. Prabhudesai, N.J. Mason, E. Krishnakumar, Eur. Phys. J. D 70, 134 (2016). DOI 10.1140/epjd/e2016-70180-y

23. P. Nag, D. Nandi, Phys. Rev. A 91, 056701 (2015). DOI 10.1103/PhysRevA.91. 056701

24. S.X. Tian, Y. Luo, Phys. Rev. A 91, 056702 (2015). DOI 10.1103/PhysRevA.91. 056702

25. A. Dora, J. Tennyson, K. Chakrabarti, Eur. Phys. J. D 70, 197 (2016)

26. L.A. Morgan, J. Tennyson, C.J. Gillan, Comput. Phys. Commun. 114, 120 (1998)

27. J.M. Carr, P.G. Galiatsatos, J.D. Gorfinkiel, A.G. Harvey, M.A. Lysaght, D. Madden, Z. Mašín, M. Plummer, J. Tennyson, Eur. Phys. J. D 66, 58 (2012)

28. J. Tennyson, Phys. Rep. 491, 29 (2010)

29. J. Tennyson, J. Phys. B: At. Mol. Opt. Phys. 29, 1817 (1996)

30. J. Tennyson, C.J. Noble, Comput. Phys. Commun. 33, 421 (1984)

31. D.T. Stibbe, J. Tennyson, Comput. Phys. Commun. 114, 236 (1998)

32. D.A. Little, J. Tennyson, M. Plummer, A. Sunderland, Comput. Phys. Commun. 215, 137 (2017). DOI 10.1016/j.cpc.2017.01.005

33. J. Tennyson, L.A. Morgan, Phil. Trans. A 357, 1161 (1999)

34. H.J. Werner, P.J. Knowles, F.R. Manby, M. Schütz, et al. Molpro, version 2006.1, a package of ab initio programs (2006). See "http://www.molpro.net"

35. E.S. Nielsen, P. Jørgensen, J. Oddershede, J. Chem. Phys. 73, 6238 (1980). DOI https://doi.org/10.1063/1.440119

36. D.T. Stibbe, J. Tennyson, J. Phys. B: At. Mol. Opt. Phys. 30, L301 (1997)

37. A. Faure, J.D. Gorfinkiel, L.A. Morgan, J. Tennyson, Comput. Phys. Commun. 144, $224(2002)$

38. A. Dora, L. Bryjko, T. van Mourik, J. Tennyson, J. Chem. Phys. 130, 164307 (2009)

39. D. Darby-Lewis, Z. Masin, J. Tennyson, J. Phys. B: At. Mol. Opt. Phys. 50, 175201 (2017). DOI 10.1088/1361-6455/aa8161 